\title{
Influencia de la naturaleza internacional de empresas peruanas en su información de sostenibilidad
}

\section{Influence of the international nature of Peruvian companies in sustainability reporting}

\author{
Julio Hernández Pajares ${ }^{1}$ \\ Recibido: 02-10-2017 - Aceptado: 12-12-2017 \\ DOI: https://doi.org/10.26441/RC17.1-2018-A4
}

\begin{abstract}
RESUMEN: Como respuesta a regulaciones promovidas por instituciones internacionales y debido a la influencia de organizaciones transnacionales, la información de sostenibilidad ha experimentado un importante desarrollo en los últimos años para las empresas latinoamericanas. En esta línea, las organizaciones peruanas también han participado en iniciativas de información bajo el modelo de la Global Reporting Initiative (GRI). Por esta razón, el objetivo del presente estudio es analizar si los factores de tamaño, naturaleza transnacional y carácter internacional de sus clientes son determinantes en el nivel de cumplimiento de la información de sostenibilidad de las empresas peruanas. Esto toma como referencia los criterios del GRI presentes en sus memorias de sostenibilidad. A partir de ello, los resultados indican que la pertenencia a grupos transnacionales y las relaciones con clientes del exterior sí se constituyen como factores determinantes en el nivel de cumplimiento de la información de los aspectos de desempeño ambiental, principalmente.
\end{abstract}

Palabras clave: América Latina; Global Reporting Initiative; información de sostenibilidad; Perú; responsabilidad social corporativa.

\begin{abstract}
In response to regulations promoted by international institutions and due to the influence of transnational organizations, sustainability reporting has experienced an important development in recent years for Latin American companies. In this line, Peruvian organizations have also participated in reporting initiatives under the model of the Global Reporting Initiative (GRI). For this reason, the aim of this study is to analyze whether the factors of size, transnational nature and international character of its customers are determinants in the level of compliance with the sustainability information of Peruvian companies. This takes as a reference the GRI criteria present in its sustainability reports. Based on this, the results indicate that belonging to transnational groups and relationships with foreign customers do constitute themselves as determining factors in the level of compliance with information on aspects of environmental performance, mainly.
\end{abstract}

Keywords: Corporate Social Responsibility; Global Reporting Initiative; Latin America; Peru; Sustainability Reporting.

1 Julio Hernández Pajares es Licenciado en Ciencias Administrativas por la Pontificia Universidad Católica del Perú, Master en Gestión Internacional de la Empresa y Doctor en Contabilidad y Finanzas por la Universidad de Zaragoza. Es profesor del Área Académica de Contabilidad y Auditoría de la Facultad de Ciencias Económicas y Empresariales de la Universidad de Piura. julio.hernandez@udep.pe, https://orcid.org/0000-0002-7481-2912 


\section{Introducción}

Diversas teorías explican el comportamiento socialmente responsable de las empresas. Al respecto, la literatura recoge distintos enfoques. En primer lugar, el económico justifica un desempeño responsable para la obtención de beneficios en las empresas. En segundo lugar, el social explica las prácticas sociales como una respuesta a la demanda legítima de los grupos de interés. En tercer lugar, el político alude al cumplimiento de obligaciones sociales de las empresas bajo el concepto de ciudadanía corporativa. Por último, el enfoque ético se refiere a la satisfacción de las demandas sociales más allá de un objetivo económico (Garrigá y Mele, 2004; Kemper y Martin, 2010).

Sobre la información de sostenibilidad, se puede asegurar que, actualmente, está vinculada con la Global Reporting Initiative (GRI), que constituye una de las instituciones más relevantes en este ámbito. No obstante, el reporte de sostenibilidad de las empresas latinoamericanas no se ha implementado de forma similar que en países desarrollados. Esto se debe a que la adopción del modelo de la GRI se ha presentado principalmente en sectores en donde los factores medioambientales y sociales tienen un mayor impacto y representan mayores riesgos, por lo que la imagen de dichas compañías está más expuesta (Marimon et al., 2012; Alonso-Almeida et al., 2015).

En el contexto latinoamericano de nuestra investigación, los estudios sobre el desempeño de la Responsabilidad Social Empresarial (RSE) señalan que ha estado dirigida a responder a los grupos de interés con los que se tiene una relación directa como trabajadores y clientes sin la implementación formal de políticas establecidas para ese fin (Vives, 2006; Jamali et al., 2009). También, es importante precisar que el presente estudio es consecuente con lo señalado por Luken y Stares (2005), Araya (2006) y Jamali et al. (2009) acerca de la importancia del desarrollo de la investigación de desempeño de la RSE en países en vías de desarrollo.

Numerosas investigaciones exponen enfoques teóricos diversos para explicar las razones de la presentación de información de RSE o sostenibilidad por las empresas como una respuesta a las expectativas de sus grupos de interés $y$, a su vez, legitimar su desempeño de sostenibilidad de acuerdo con los valores de la sociedad. (Adams, 2002; Parker, 2005). Asimismo, se ha concluido que dicha información en empresas latinoamericanas responde a una influencia institucional de socios comerciales de países desarrollados y organizaciones no gubernamentales transnacionales que incentivan la divulgación de aspectos ambientales y sociales (Araya, 2006; Perez-Batres et al., 2010; Calixto, 2013).

En el Perú, las prácticas de RSE son adoptadas de forma voluntaria como las promovidas por asociaciones empresariales o asociaciones sin fines de lucro. Un claro ejemplo de ello es la labor realizada por la asociación Perú 2021 que busca incentivar las prácticas de RSE en el empresariado peruano según estándares de organizaciones internacionales (Calixto, 2013). 
Vinculado con ello, la mayor apertura a mercados internacionales en la última década ha obligado a las empresas peruanas a ampliar su desempeño con sus distintos grupos de interés, dirigido a inversiones sociales orientadas a establecer mejores relaciones con sus comunidades. Un caso representativo, como señalan Quiroz (2009) y Nakasone (2015), se refiere a las empresas mineras, lo que ha generado prácticas de desempeño e información social y ambiental.

Dicho todo esto, se puede deducir que la influencia institucional externa de organizaciones como la GRI o el Pacto Mundial de la ONU, la pertenencia a grupos internacionales y las relaciones con partes comerciales internacionales determinan el desempeño e información de sostenibilidad, en lugar de la promoción y regulación del Estado, poco o nada difundidas en países en vías de desarrollo (Cetindamar y Husoy, 2007; Perez-Batres et al., 2010; Alonso-Almeida et al., 2015).

Después de analizar los aportes presentados, es más claro el cuestionamiento de si la naturaleza y prácticas de información de sostenibilidad por parte de las empresas peruanas también obedecen a una influencia de sus clientes internacionales o la pertenencia a grupos internacionales y por lo tanto con mayores recursos y tamaño. En ese sentido, el objetivo del presente estudio es determinar si los factores como el tamaño, naturaleza transnacional y naturaleza internacional de sus clientes afectan el nivel de cumplimiento de información de sostenibili- dad, de acuerdo con los criterios de la GRI para las empresas peruanas que han publicado sus memorias por el periodo del 2006-2015.

Este artículo comprende, además de esta introducción, una segunda parte que presenta antecedentes teóricos sobre la información de sostenibilidad y el planteamiento de las hipótesis. La tercera parte explica el diseño de la investigación cuantitativa; la cuarta, el análisis de los resultados; la quinta, la discusión de los resultados con respecto a las hipótesis; y, finalmente, se exponen las conclusiones.

\section{Marco teórico}

Uno de los principios de la RSE en las empresas es el de transparencia. Esto significa que la relación con los grupos de interés obliga a rendir cuenta respecto al comportamiento responsable de las organizaciones (AECA, 2004). Se debe considerar, entonces, que la información de RSE es una de las consecuencias de la extensión y aceptación del concepto de desarrollo sostenible en las organizaciones (Gray et al., 1996; Moneva et al., 2006). Para comprender mejor la noción de transparencia, se implica el concepto de accountability que supone la responsabilidad de emprender ciertas acciones y la de rendir cuenta sobre las mismas (Gray et al., 1996).

\section{1. Influencia institucional interna- cional}

Ya se había mencionado que iniciativas de información de organizaciones internacionales, como el GRI y el Pacto 
Mundial de las Naciones Unidas, han tenido una gran influencia en países desarrollados. Estas han surgido con el fin de promover prácticas de desempeño y transparencia de sostenibilidad como parte de la operación y estrategia de los negocios y así obtener ventajas competitivas como una oportunidad de crear valor en las empresas y para la sociedad (Jamali, 2007). Sin embargo, algunos estudios precedentes señalan también que este modelo mundial de divulgación de sostenibilidad de la GRI no ha sido el más apropiado para la revelación de actividades sociales y ambientales propias de empresas de países en vías en desarrollo que difieren por el entorno donde se desarrollan (Waddock, 2008; Marimon, et al., 2012). Asimismo, según lo planteado por Alonso-Almeida et al. (2015), en el caso de la mayoría de empresas de países latinoamericanos, el desarrollo de su información de sostenibilidad ha presentado un crecimiento moderado y desigual para cada país y tipo de industria.

A pesar de que las iniciativas de reporte de sostenibilidad a nivel internacional han seguido la mencionada propuesta, la información no muestra siempre una relación entre el desempeño y el compromiso con los grupos de interés y con los supuestos de desarrollo sostenible, sino un interés de legitimidad del desempeño sostenible. Es así que, siguiendo las opiniones de Deegan (2002), Moneva et al. (2006) y Husillos (2007), esta divulgación se refiere a actuaciones positivas que pueden influenciar en las opiniones externas sobre su organización con sus grupos de interés con mayor impacto.

Las empresas latinoamericanas que más desarrollo han tenido en las prácticas de desempeño e información de RSE corresponden a las empresas subsidiarias de grupos transnacionales y las que realizan actividad de exportación con países desarrollados como en el caso de otros países en vías de desarrollo (Islam y Deegan, 2010; Momin y Parker, 2013). Esto quiere decir que son aquellas con mayores relaciones transnacionales, quienes presentan una práctica e información de sostenibilidad más destacada con el fin de mejorar su legitimidad frente a sus socios comerciales internacionales, pertenecientes a la Unión Europea, por ejemplo, donde la promoción del desarrollo sostenible es una política pública. Esto significaría, entonces, que no es un cumplimiento normativo el que dirige las prácticas de sostenibilidad en las empresas latinoamericanas, sino una influencia de organizaciones y socios externos (Cetidamar y Husoy, 2007; Perez-Batres et al., 2010, Calixto, 2013; Alonso-Almeida et al., 2015). Finalmente, vale la pena destacar las prácticas las empresas que poseen mayor influencia en los grupos de interés debido a su actividad de explotación de recursos naturales o industriales (Delmas y Toffel, 2008; Marimon et al., 2012; Alonso-Almeida et al., 2015).

Consecuentemente, se puede confirmar que la divulgación en memorias de sostenibilidad en el Perú no solo responde a una influencia externa, sino que ha estado dirigida a la búsqueda de 
una reputación social como indica Porro (2008); de publicar lo "políticamente correcto" del desempeño, como señala Tostes y Chero (2010) o de justificar su desempeño con sus grupos de interés con mayor impacto social (Jaskoski, 2014; Helwege, 2015).

\section{2. Otros factores determinantes de información}

$\mathrm{Al}$ respecto, existen investigaciones sobre los factores que determinan el nivel de divulgación de RSE o sostenibilidad. Éstas señalan que el tipo de industria y tamaño son los más determinantes en el nivel de transparencia. Esto justifica el hecho de que las empresas de países desarrollados de mayor tamaño y con mayor impacto ambiental y social presentan un mayor nivel de información (Moneva y Llena, 1996; Archel, 2003; Llena et al., 2007; Reverte, 2009; Gamerschlag et al., 2011). De la misma manera ocurre con empresas en vía de desarrollo (Amran y Haniffa, 2011; Ali et al., 2017; Ortas y Moneva, 2011; Alonso-Almeida et al., 2015; Rabasedas et al., 2016).

Los informes de sostenibilidad de empresas latinoamericanas han tenido un importante crecimiento en los últimos años, debido a influencia de organizaciones no gubernamentales e influencia de grupos de interés, como señalan ACCA (2004) y Marimon et al. (2012). Por otro lado, algunos estudios confirman que las empresas con un significativo impacto ambiental, de gran tamaño y con cotización pública son las que presentan una mayor divulgación de sostenibilidad (Perez Batres et al.,
2010; Alonso-Almeida et al., 2015).

Como se señaló con anterioridad, un aspecto relevante que explica el nivel de información de sostenibilidad es la influencia institucional de las empresas principales de grupo y organizaciones transnacionales como señalan Araya (2006), Chen y Bouvain (2009), PerezBatres et al. (2010) y Fifka (2013). Según Jenkins y Yakovleva (2016), este hecho se comprueba, específicamente, en el caso de empresas mineras transnacionales, con grandes recursos y tamaño y con una amplia experiencia de divulgación.

En el Perú, el nivel de información de sostenibilidad de las empresas ha aumentado, principalmente, para las empresas con mayor impacto ambiental y social, como es el caso de las empresas mineras y de explotación de petróleo como señalan Porro (2008) y Nakasone (2015) y con mayor impacto ambiental y social como es el caso de las empresas de mayor tamaño y explotación de recursos naturales y energía y con mayor responsabilidad con los productos que exportan, como las empresas agroindustriales (AlonsoAlmeida, 2015; Hernández, 2016).

De acuerdo con las propuestas teóricas anteriores, se plantean las siguientes hipótesis:

H1: El tamaño no tiene una influencia significativa en el nivel de cumplimiento de la información de desempeño ambiental, laboral, responsabilidad de productos y social

H2: El tamaño, la naturaleza transnacional de la empresa, la naturaleza internacional de los clientes, no son factores 
determinantes en los niveles de información de desempeño ambiental y social.

\section{Metodología}

La presente investigación es del tipo cuantitativo, con alcance de tipo explicativo y correlacional. El modelo de investi- gación busca contrastar si las variables independientes de tamaño de las empresas, naturaleza transnacional y carácter internacional de los clientes influyen en el nivel de cumplimiento de información de sostenibilidad como variable dependiente, como observamos en el Gráfico 1.

Grafico 1. Modelo Teórico de Estudio

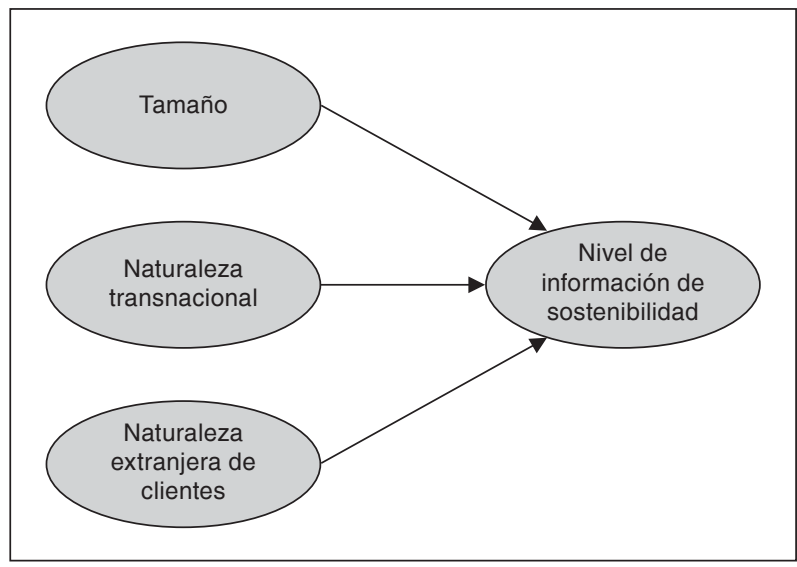

Fuente: Elaboración propia

Para la obtención de las variables del nivel de cumplimiento de información, se ha aplicado la metodología de análisis de contenido de reportes de sostenibilidad publicados por empresas peruanas, aplicada en estudios como los de Gray et al. (1995); Llena et al. (2007); Moneva et al. (2007) y Sweeney y Coughlan (2008).

$\mathrm{El}$ análisis del contenido se ha realizado sobre los informes de sostenibilidad y RSE publicados en la base de da- tos de la GRI, con respecto al nivel de cumplimiento del principio de materialidad para los indicadores principales de desempeño ambiental y social de acuerdo con las Guías G3 y G4 de la GRI (2006; 2013). Se han estudiado las 67 empresas con fines de lucro, que han publicado alguna vez en la Sustainability Disclosure Database de la $\mathrm{GRI}^{2}$, para sus más recientes publicaciones de sus memorias de sostenibilidad, entre los años 2006 y 2015.

2 Global Reporting Initiative, Sustainability Disclosure Database, acceso en http://database.globalreporting.org/ search. Consultado el 30 de marzo de 2017. 
La evaluación del nivel de cumplimiento se ha llevado a cabo mediante una escala de Likert sobre 20 variables cuantitativas basadas en los indicadores principales de desempeño de sostenibilidad (ver Anexo 1). En este se consideró el principio de materialidad de la Guía del GRI (2006, 2013), en la información sobre los indicadores de desempeño ambiental y social (laboral, responsabilidad de productos y sociedad) de la memoria más reciente publicada. Además, el criterio de materialidad se evaluó sobre los impactos significativos, sociales, ambientales y económicos de la organización o aquellos que podrían ejercer una influencia sustancial en decisiones de los grupos de interés recogidos en los indicadores de la Guía de la GRI (GRI, 2006, 2103).

Las variables independientes consideradas fueron tres. En primer lugar, figura el tamaño para el cual se tiene en cuenta el número de trabajadores. Según la clasificación de MERCOSUR y Ley MYPE ${ }^{3}$, una empresa es pequeña cuando su número de trabajadores está comprendido hasta 50 trabajadores; mediana, hasta 100 trabajadores y grande, más de 100 trabajadores. Además, se verifica el volumen de ventas del periodo estudiado y señalado en sus memorias. En segundo lugar, se atiende a la naturaleza transnacional, si pertenece a un grupo transnacional o no. Por último, es importante observar si presentan o no clientes con quienes realizan actividades de exportación.

Se aplicaron herramientas de análisis multivariante correspondientes al análisis factorial para reducir las variables a componentes que expliquen la actividad de información sostenibilidad, pruebas ANOVA, así como de regresión lineal para determinar la significatividad de la influencia de los factores en el nivel de información.

\section{Análisis de resultados}

\section{1. Análisis descriptivo}

Las empresas peruanas que publican sus informes de sostenibilidad en la base de datos de la GRI han presentado un crecimiento significativo del número de publicaciones en los últimos años como se muestra en el Gráfico 2. De hecho, el número de publicaciones desde el 2006 es importante, principalmente, para empresas del sector minero e industrial. Sin embargo, en el 2016, se observa una pequeña disminución debido, probablemente, al decrecimiento económico del país, postergaciones de proyectos mineros y/o menores inversiones extranjeras.

El mayor número de publicaciones corresponde a las empresas mineras, industriales y de energía. Esto obedece a que la actividad productiva de las empresas mineras tiene una fuerte influencia e impacto en las comunidades campesinas y en el medioambiente donde se desarrollan estas colectividades. Por ello, se presenta una necesidad

3 Ley de Promoción de la Competitividad, Formalización y Desarrollo de la Micro y Pequeña Empresa y del Acceso al Empleo Decente. De acuerdo con Norma DS N007-2008-TR 
de rendir cuenta sobre una actividad responsable, frente al Estado, la sociedad y las organizaciones civiles como una búsqueda de legitimidad social de sus actividades (Gifford y Kestler, 2008; Slack, 2012; Jaskoski, 2014).

Gráfico 2. Número de publicaciones anules de memorias según sector

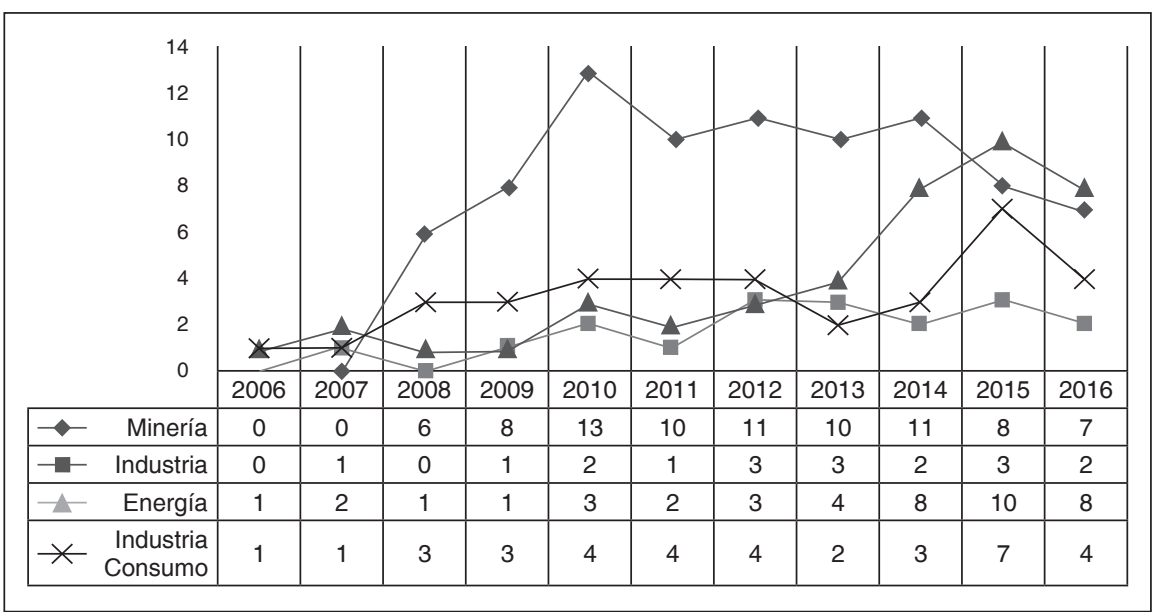

Fuente: Elaboración propia basado en la Sustainability Disclosure Database. Abril de 2017.

Cabe resaltar que la frecuencia de publicación no ha sido periódica, no todas las empresas han publicado información anual como señalan Calixto (2013) y Hernández (2016). Casi el 50\% de las empresas solo ha publicado dos memorias de sostenibilidad y cerca del $10 \%$ han realizado publicaciones periódicas anuales, como se observa en el Gráfico 3. Debido a esto, la información no describe control y gestión con respecto a periodos anteriores de objetivos de sostenibilidad con sus grupos de interés.

Las empresas que publican periódicamente (más de dos publicaciones) sus memorias tienen un adecuado nivel de información de gestión y grupos de interés, realizan un seguimiento de los objetivos estratégicos de sostenibilidad y, además, presentan un importante desempeño ambiental. Entre ellas se encuentran empresas financieras, mineras, industriales y de telefonía, transnacionales, principalmente. Aquellas con menor número de memorias publicadas, en su mayoría empresas industriales y de servicios, solo destacan en la información de desempeños laborales y sociales con la comunidad. 
Gráfico 3. Frecuencia de Publicaciones de Memorias

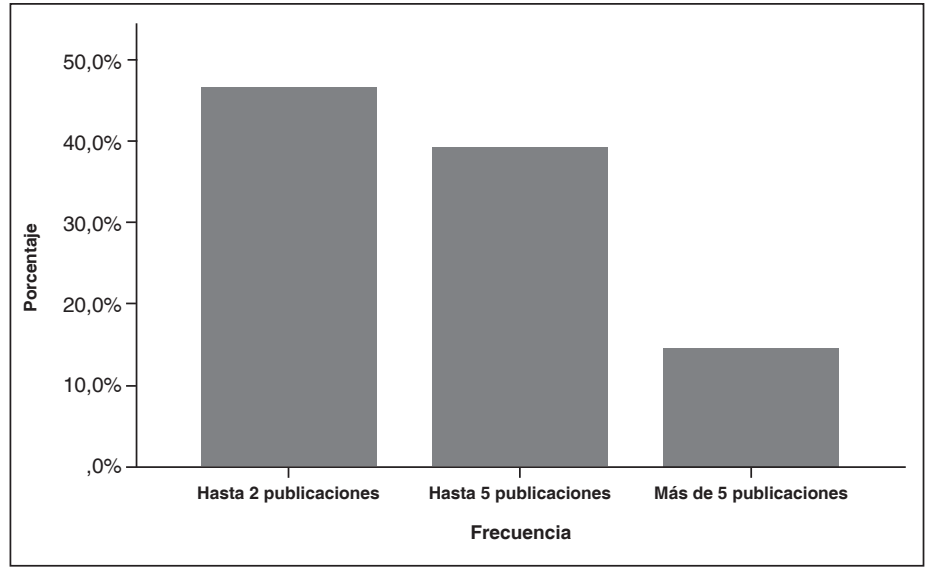

Fuente: Elaboración propia

\section{2. Análisis Correlacional}

Para el estudio cuantitativo, se procedió con la prueba de análisis factorial para reducción de las 20 variables de cumplimiento de prácticas ambientales y sociales. Como resultado, se obtuvieron cuatro factores representativos del nivel de cumplimiento de información de sostenibilidad. La prueba resultó confiable debido a que se obtuvo un estadístico de 0,754 de adecuación maestral y una significatividad de 0 (sig.<0.05) de rechazo de hipótesis nula de independencia de variables según pruebas de Kaiser-Meyyer-Olkin y Bartlett respectivamente.

Según las variables de sector empresarial (Gráfico 4) y tamaño (Gráfico 5), los componentes presentan distintos niveles de información.

El primer componente denominado "factor ambiental" representa las prácticas según los indicadores ambientales relacionados con la gestión de agua, residuos, emisiones, energía. Es característico y con mayor incidencia en las empresas mineras, así como en las industriales y de energía, ya que, por su naturaleza, utilizan recursos naturales y rinden cuenta de ello. Además, el segundo componente de "factor laboral" de prácticas con los colaboradores, es ampliamente informado por las empresas financieras y de seguros. Las mineras y de servicios (constructoras y telefonía), además de su desempeño ambiental, realizan importantes prácticas de desempeño con sus colaboradores. El tercer componente de "factor de seguridad de productos" representa las prácticas según los indicadores de responsabilidad de productos con los clientes como las prácticas informadas de seguridad e información de productos de consumo o exportación de empresas industriales y agrícolas. Por 
último, el cuarto componente de "desempeño social" con la comunidad es aplicado en gran medida por todas las empresas, pero destaca el sector financiero, sobre todo, aquellas adscritas al Pacto Mundial de la ONU.

Gráfico 4. Componentes del análisis factorial según sector empresarial

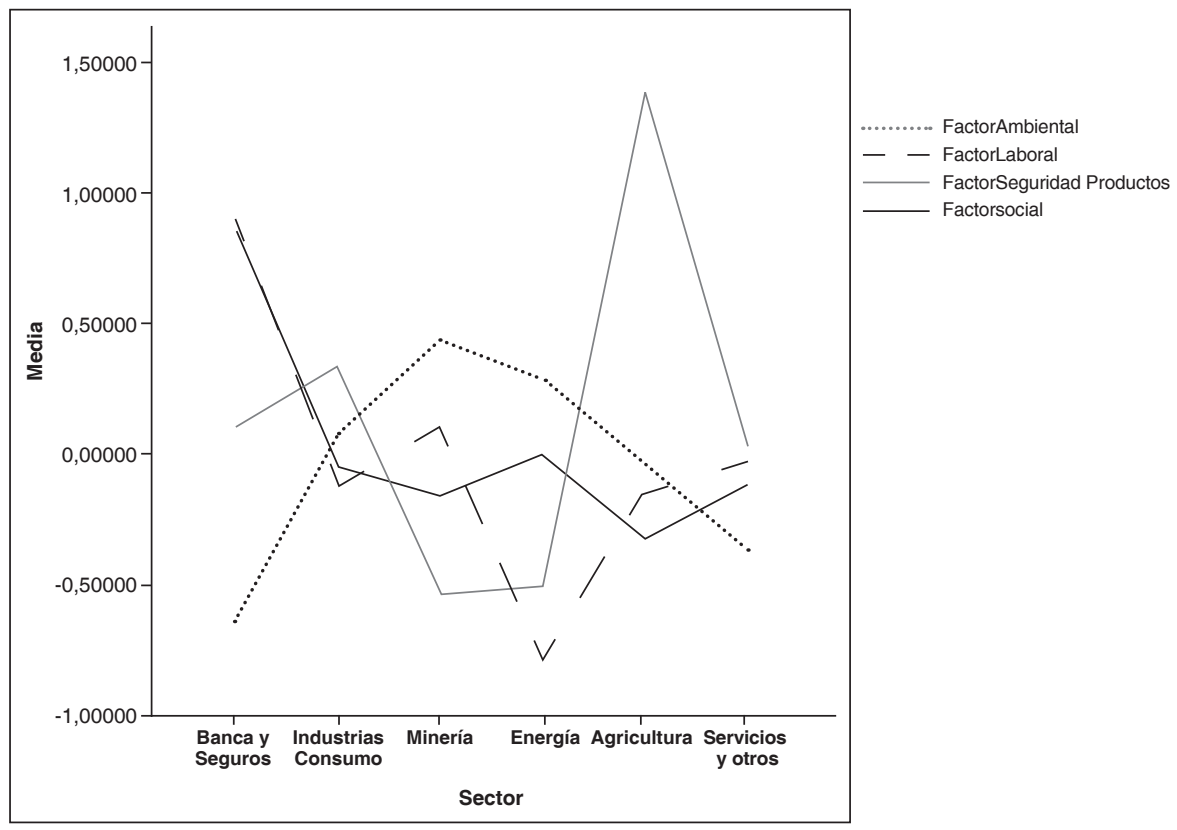

Fuente: Elaboración propia

Se observa también que las empresas más grandes y con mayor número de colaboradores destacan en las prácticas de sostenibilidad divulgadas, en desempeños sociales y de responsabilidad de productos.

Para evaluar la significatividad de las diferencias en el nivel de información por sector empresarial, se realizaron las pruebas ANOVA de un factor para los cuatro componentes, para evaluar si la media del nivel de información varía significativamente para las empresas según su tamaño. 
Gráfico 5. Componentes del análisis factorial según sector empresarial

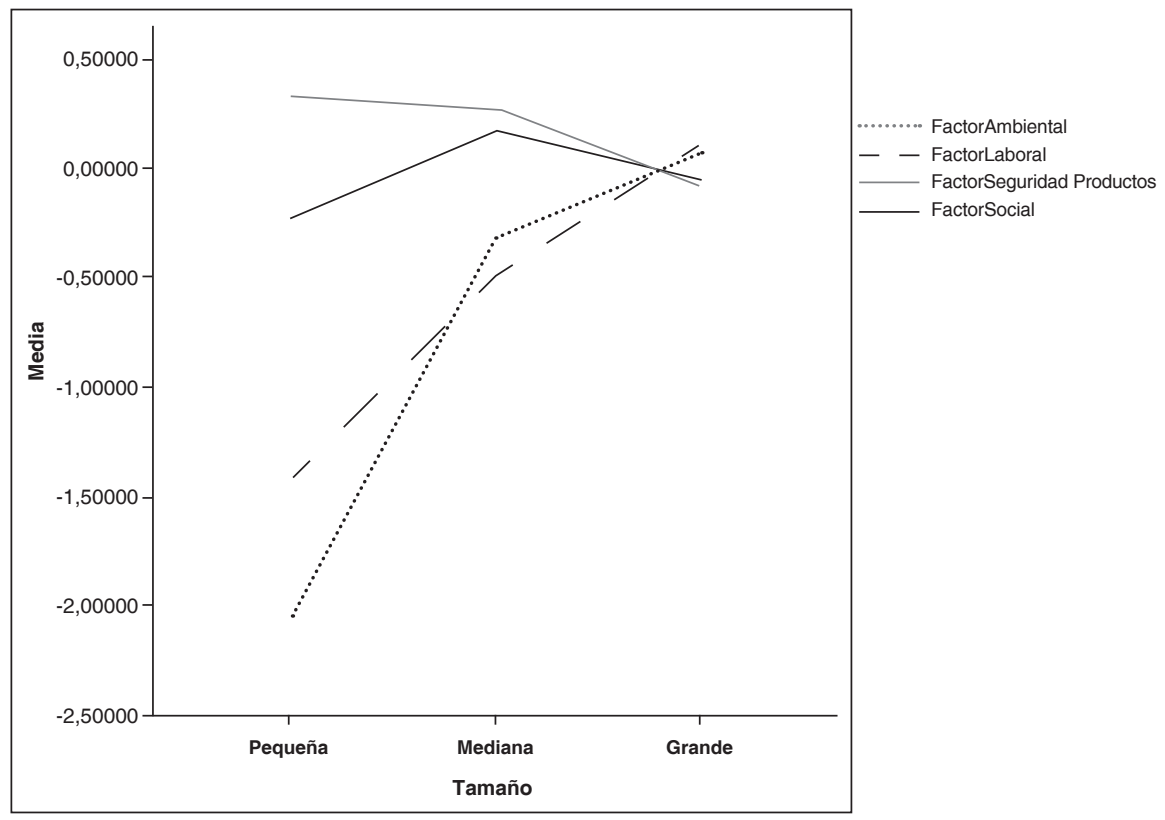

Fuente: Elaboración propia

Tabla 1. Resultados prueba ANOVA de componentes según tamaño

\begin{tabular}{|l|l|r|r|r|r|r|}
\hline \multicolumn{2}{|l|}{ Factores } & $\begin{array}{c}\text { Suma de } \\
\text { cuadrados }\end{array}$ & gl & $\begin{array}{c}\text { Media } \\
\text { cuadrática }\end{array}$ & F & Sig. \\
\hline \multirow{4}{*}{ Factor Ambiental } & Entre grupos & 4,301 & 2 & 2,151 & 2,229 &, 116 \\
\cline { 2 - 8 } & Dentro de grupos & 62,699 & 65 &, 965 & & \\
\cline { 2 - 8 } & Total & 67,000 & 67 & & & \\
\hline \multirow{4}{*}{ Factor Laboral } & Entre grupos & 9,920 & 2 & 4,960 & 5,648 &, 005 \\
\cline { 2 - 8 } & Dentro de grupos & 57,080 & 65 &, 878 & & \\
\cline { 2 - 8 } & Total & 67,000 & 67 & & & \\
\hline Factor Seguridad & Entre grupos &, 594 & 2 &, 297 &, 291 &, 749 \\
\cline { 2 - 8 } & Dentro de grupos & 66,406 & 65 & 1,022 & & \\
\cline { 2 - 8 } & Total & 67,000 & 67 & & & \\
\hline Factor Social & Entre grupos &, 320 & 2 &, 160 &, 156 &, 856 \\
\cline { 2 - 8 } & Dentro de grupos & 66,680 & 65 & 1,026 & & \\
\cline { 2 - 7 } & Total & 67,000 & 67 & & & \\
\hline
\end{tabular}


La Tabla 1 muestra que el tamaño incide significativamente (sig. $<0.05$ ) solo en el nivel de información de desempeño laboral. Es decir, las empresas con mayor número de colaboradores presentan un mayor nivel de cumplimiento de información sobre desempeño laboral. Para el resto de componentes, el tamaño no incide significativamente en el nivel de cumplimiento de información de sostenibilidad.

Se realizó una regresión lineal para evaluar si el tamaño (ventas), la naturaleza transnacional y la naturaleza exportadora (como variables independientes) influyen significativamente en el nivel de información de sostenibilidad (considerando los aspectos ambiental y social) según este modelo:

$$
y=b_{0}+b_{1} \cdot x_{1}+b_{2} \cdot x_{2}+b_{3} \cdot x_{3}+u
$$

Dónde:

y: nivel de cumplimiento de información de sostenibilidad (ambiental o social)

$\mathrm{x}_{1}$ : tamaño

$\mathrm{x}_{2}$ : naturaleza transnacional

$\mathrm{x}_{3}$ : naturaleza exportadora

$u$ : constante

Para validar el modelo de regresión lineal, considerando el nivel de información ambiental como variable dependiente, se verifica un contraste de regresión de Sig. $0.01<0.05$ como muestra la Tabla 2. Estos indicadores validan que las variables independientes influyen con suficiencia en la variable independiente.

Tabla 2. Indicadores de Validación de Regresión

\begin{tabular}{|c|r|r|r|r|r|r|}
\hline Modelo & $\begin{array}{c}\text { Suma de } \\
\text { cuadrados }\end{array}$ & gl & Media cuadrática & F & Sig. \\
\hline & Regresión & 14,977 & 3 & 4,992 & 6,142 &, $001^{\mathrm{b}}$ \\
\hline Residuo & 52,023 & 64 &, 813 & & \\
\hline Total & 67,000 & 67 & & & \\
\hline
\end{tabular}

a. Variable dependiente: FactorAmbiental

b. Predictores: (Constante), Exportadora, Ventas en MM S/, Transnacional

Fuente: Elaboración propia

Los resultados de la regresión en la Tabla 3 demuestran que las variables tienen incidencia significativa (Sig. <0.05) sobre el nivel de información ambiental. Son las que expli- can el carácter transnacional y la naturaleza exportadora de las empresas las que influyen en el nivel de cumplimiento de información de este tipo. 
Tabla 3. Estadísticos Regresión Lineal para el Componente Ambiental

\begin{tabular}{|c|c|c|c|c|c|}
\hline \multirow[t]{2}{*}{ Modelo } & \multicolumn{2}{|c|}{$\begin{array}{l}\text { Coeficientes no } \\
\text { estandarizados }\end{array}$} & \multirow{2}{*}{\begin{tabular}{|c|}
$\begin{array}{c}\text { Coeficientes } \\
\text { estandarizados }\end{array}$ \\
Beta \\
\end{tabular}} & \multirow[t]{2}{*}{$\mathbf{t}$} & \multirow[t]{2}{*}{ Sig. } \\
\hline & B & Error estándar & & & \\
\hline (Constante) &,- 552 & 170 & & $-3,257$ & ,002 \\
\hline Ventas en MM S/ & $2,342 E-5$ & ,000 & , 145 & 1,279 & ,206 \\
\hline Transnacional & ,519 & 230 & ,260 & 2,254 & ,028 \\
\hline Exportadora &, 538 & ,227 & ,270 & 2,373 & ,021 \\
\hline
\end{tabular}

a. Variable dependiente: FactorAmbiental

Fuente: Elaboración propia

También, se realizó una regresión lineal para evaluar si el tamaño (ventas), la naturaleza transnacional y la naturaleza exportadora (como variables independientes) influyen significativamente en el nivel de información social. Para validar el mo- delo, se verificó un contraste de regresión de Sig. 0.360 no menor a 0.05 como muestra la Tabla 4. Estos indicadores señalan que las variables independientes no influyen de manera significativa en dicha variable de información ambiental.

Tabla 4. Indicadores de Validación de Regresión

\begin{tabular}{|c|c|c|c|c|c|}
\hline Modelo & $\begin{array}{c}\text { Suma de } \\
\text { cuadrados }\end{array}$ & gl & $\begin{array}{c}\text { Media } \\
\text { cuadrática }\end{array}$ & $\mathbf{F}$ & Sig. \\
\hline Regresión & 3,255 & 3 & 1,085 & 1,089 &, $360^{b}$ \\
\hline Residuo & 63,745 & 64 & ,996 & & \\
\hline Total & 67,000 & 67 & & & \\
\hline
\end{tabular}

a. Variable dependiente: FactorSocial

b. Predictores: (Constante), Exportadora, Ventas en MM S/, Transnacional

Fuente: Elaboración propia

Tabla 5. Estadísticos Regresión Lineal para el Componente Social

\begin{tabular}{|c|c|c|c|c|c|}
\hline \multirow{2}{*}{ Modelo } & \multicolumn{2}{|c|}{$\begin{array}{l}\text { Coeficientes no } \\
\text { estandarizados }\end{array}$} & \multirow{2}{*}{\begin{tabular}{|c|}
$\begin{array}{c}\text { Coeficientes } \\
\text { estandarizados }\end{array}$ \\
Beta
\end{tabular}} & \multirow[t]{2}{*}{ t } & \multirow{2}{*}{ Sig. } \\
\hline & B & Error estándar & & & \\
\hline (Constante) &,- 028 & ,188 & & - -149 & ,882 \\
\hline Ventas en MM S/ & $3,394 \mathrm{E}-5$ & ,000 & 211 & 1,674 & 099 \\
\hline Transnacional & ,031 & ,255 & ,016 & ,123 & ,903 \\
\hline Exportadora &,- 194 & 251 &,- 097 &,- 773 & ,443 \\
\hline
\end{tabular}

a. Variable dependiente: FactorSocial 
Los resultados de la regresión en la Tabla 5 para el componente social confirman que el tamaño, carácter transnacional y exportadora de las empresas no tienen incidencia significativa en la variable independiente de nivel de información social.

\section{Discusión}

Respecto a los factores analizados, si las empresas estudiadas pertenecen a un grupo transnacional, poseen clientes del exterior y tienen un mayor tamaño, no se encuentra en todos los casos, que exista una influencia en todos los aspectos de información de sostenibilidad.

La prueba de análisis de varianza (ANOVA), encuentra que la media del nivel de información de sostenibilidad no difiere significativamente en todos sus aspectos de acuerdo al tamaño de las empresas estudiadas, solo el factor de nivel de información laboral varía significativamente con el tamaño de las empresas. Es así que la H1, se rechaza solo para el caso de la información de desempeño laboral, que se ve influenciada por el tamaño de las empresas según el número de trabajadores.

Se encuentra que las empresas, especialmente las financieras, mineras y de servicios, y con mayor número de colaboradores presentan un mayor nivel de cumplimiento de información sobre desempeño laboral. Son destacadas en las memorias de estas empresas, el nivel de información sobre detalle de sus trabajadores y prácticas sobre composición de los colaboradores, seguridad laboral y actividades de formación y capacitación (Perez-Batres et al., 2010; Alonso-
Almeida et al., 2015; Rabasedas et al., 2016).

Con respecto a la influencia internacional (pertenencia a grupos transnacionales y actividades de exportación con clientes del exterior), el análisis de regresión lineal los encuentra como factores determinantes en el nivel de información ambiental por lo que se rechaza la hipótesis H2 es ese aspecto.

Las empresas que pertenecen a grupos transnacionales reciben influencia en los niveles de reporte de sostenibilidad corporativo en los aspectos ambientales, principalmente, como es el caso de las empresas mineras, energía e industriales. Esto les permite una experiencia de publicación y una influencia institucional de sus principales empresas extranjeras en las políticas de reporte (Chen y Bouvain, 2009; Perez Batres et al., 2010; Ortas y Moneva, 2011; Fifka, 2013; Alonso-Almeida et al., 2015; Rabasedas et al., 2016).

Las empresas agroindustriales, manufactureras de productos de consumo y mineras que realizan actividad de exportación valoran el desempeño e información de sostenibilidad en los aspectos ambientales, debido a que los clientes del exterior exigen dichos estándares. Esto explica, desde el punto de vista institucional, que los clientes influyen significativamente en las prácticas ambientales y certificaciones de los productos exportados (Araya, 2006; Delmas y Toffel, 2008; Marimon et al., 2012; Alonso-Almeida et al., 2015).

El resultado de la regresión lineal no concluye que la pertenencia a grupos transnacionales o la naturaleza expor- 
tadora de las empresas tengan influencia significativa en el nivel de información social, por lo que no se rechaza la segunda hipótesis (H2) para la variable independiente de desempeño social.

No es el carácter transnacional ni la naturaleza exportadora de las empresas, los principales influyentes en el nivel de cumplimiento de información de desempeño social. Este debe ser explicado, como se ha descrito en el marco teórico, por el interés de legitimar las actuaciones sociales ante la sociedad o responder a las expectativas de sus grupos de interés bajo la teoría de los stakeholders (Marimon et al., 2012). Esta situación es propia de las empresas mineras (Jaskoski, 2014; Helwege, 2015).

\section{Conclusiones}

La práctica de divulgación de sostenibilidad, en los últimos 10 años, para la mayoría de empresas peruanas, no ha sido uniforme y ha sido distinta según el tamaño y la pertenencia a un grupo transnacional que influye significativamente en las políticas de sostenibilidad, es el caso de grandes empresas mineras e industriales con desempeño e información laboral importante y las empresas transnacionales y exportadoras en su mayor información de desempeño ambiental.

Los resultados no indican en la mayoría de casos un nivel significativo de determinación de los factores estudiados en la divulgación social y ambiental, por lo que otros aspectos deben explicar el nivel de información de sostenibilidad. El estudio encuentra que muchas empresas usan la información para divulga- ción de aspectos positivos de desempeño de sostenibilidad. Constantemente, puede presentarse una búsqueda de legitimación y mejora de reputación social en la información (Deegan, 2002; Gifford y Kestler, 2008; Marimon et al., 2012).

Las empresas mineras son un caso particular. La influencia de las comunidades campesinas, las reclamaciones de impactos ambientales de poblaciones cercanas a los centros mineros o el cumplimiento de requerimientos del Estado obligan a una mayor divulgación ambiental y social que justifique y permita legitimar su desempeño (Slack, 2012; Jaskoski, 2014; Helwege, 2015).

Se observa una importante divulgación de empresas agroindustriales y de producción de consumo, que orientan sus enfoques y prácticas de sostenibilidad a sus clientes del exterior e informan sobre la gestión de sus productos, en calidad, producción limpia, salud y certificaciones (Araya, 2006; AlonsoAlmeida et al., 2015). Asimismo, existe una importante influencia de empresas con las que operan comercialmente o de sus matrices transnacionales (PerezBatres et al., 2010; Fifka, 2013).

Estos resultados también implican que el desempeño e información de sostenibilidad en empresas de países en vías de desarrollo no ha encontrado un interés similar que, en países desarrollados, probablemente debido a la falta de promoción por la sociedad civil y el Estado. Tal situación es contraria al entorno de la Unión Europea donde existe un fuerte desarrollo normativo y regulación institucional (Jamali, 2007; Perez-Batres et al., 2010; Marimon et al., 2012). 
Las implicancias de este estudio refieren una necesidad de investigar otros factores como el enfoque estraté- gico o búsqueda de legitimidad de la gerencia, respecto de la información de sostenibilidad.

\section{Anexo 1}

Indicadores de la GRI consideradas como variables de cumplimiento de desempeño de sostenibilidad

\section{Indicadores de Desempeño Económico}

EC1 Valor Económico directo generado y distribuido

\section{Indicadores de Desempeño Ambiental}

EN1 Materiales consumidos en volumen y valorizados

EN2 Consumo directo de energía desglosados por fuentes

EN3 Captación y ahorro de agua

EN4 Impactos en biodiversidad

EN5 Emisiones directas e indirectas de gases efecto invernadero y otros

EN6 Vertimiento de aguas residuales

EN7 Residuos gestionados

EN8 Iniciativas para mitigar impacto ambiental

\section{Indicadores de Desempeño Social} Indicadores de Desempeño Laboral

LA1 Detalle de Empleados

LA2 Seguridad y salud laboral

LA3 Absentismo, enfermedades, muertes relacionados con el trabajo

LA4 Horas de Formación de empleados

Indicadores de Desempeño Derechos Humanos e Impacto Social

SO1 Distribuidores y contratistas analizados en derechos humanos

SO2 Políticas de no discriminación

SO3 Programas y prácticas de impacto en la comunidad

SO4 Medidas anticorrupción

\section{Indicadores de Responsabilidad de Productos}

SO5 Impacto en seguridad y salud de clientes

SO6 Información normada para productos

SO7 Programas de cumplimiento de leyes y adhesión a códigos voluntarios de marketing 


\section{Bibliografía}

Adams, C. A. (2002). Internal organisational factors influencing corporate social and ethical reporting: Beyond current theorising. Accounting, Auditing \& Accountability Journal, 15(2), 223-250.

Ali, W., Frynas, J., y Mahmood, Z. (2017). Determinants of Corporate Social Responsibility (CSR) Disclosure in Developed and Developing Countries: A Literature Review. Corporate Social Responsibility and Environmental Management, 24(4), 273-294.

Amran, A. y Haniffa, R. (2011). Evidence in development of sustainability reporting: a case of a developing country. Business Strategy and the Environment, 20(3), 141-156.

Archel, P. (2003). La divulgación de la información social y medioambiental de la gran empresa española en el período 1994-1998: situación actual y perspectivas. Revista Española de Financiación y Contabilidad, 117, 571-601.

Araya, M. (2006). Exploring Terra Incognita: Non-financial Reporting in Corporate Latin America. Journal of Corporate Citizenship, 21, 25-38.

Alonso-Almeida, M., Marimon, F., y Llach, J. (2015). Difusión de las memorias de sostenibilidad en Latinoamérica: análisis territorial y sectorial. Estudios Gerenciales, 31(135), 139-149.

Asociación Española de Contabilidad y Administración de Empresas (AECA) (2004). Marco conceptual de la responsabilidad social corporativa, Madrid: AECA.

Association of the Chartered Certified Accountants (ACCA). 2004. Towards transparency: progress on global sustainability reporting 2004, ACCA. London: Business for Social Responsibility.

Calixto, L. (2013). A divulgação de relatórios de sustentabilidade na América Latina: um estudo comparativo. Revista de Administração, 48(4), 828-842.

Chen, S. y Bouvain, P. (2009). Is corporate responsibility converging? A comparison of corporate responsibility reporting in the USA, UK, Australia, and Germany. Journal of Business Ethics, 87, 299-317.

Cetindamar, D. y Husoy, K. (2007). Corporate Social Responsibility Practices and Environmentally Responsible Behavior: The Case of the United Nations Global Compact. Journal of Business Ethics, 76, 163-176.

Da Silva, S. y Aibar, B. (2011). Factores determinantes del grado de información medioambiental divulgada en las grandes empresas que operan en Portugal: un análisis univariante. Base, 8(1), 3-19.

Deegan, C. (2002). Introduction: The legitimising effect of social and environmental disclosures-a theoretical foundation. Accounting, Auditing \& Accountability Journal, 15(3), 282-311.

Delmas, M. y Toffel, M. (2008). Organizational responses to environmen-tal demands: opening the black box. Strategic Management Journal, 29(10), 1027-1055.

Fifka, M. (2013). Corporate responsibility reporting and its determinants in comparative perspective: a review of the empirical literature and a meta-analysis. Business Strategy and the Environment, 22(1), 1-35. 
Gamerschlag, R., Möller, K., \& Verbeeten, F. (2011). Determinants of voluntary CSR disclosure: empirical evidence from Germany. Review of Managerial Science, 5(2-3), 233-262.

Garriga E., Melé D. (2004). Corporate social responsibility theories: Mapping the territory. Journal of Business Ethics, 53: 51-71.

Gifford, B. y Kestler, A. (2008). Toward a theory of local legitimacy by MNEs in developing nations: Newmont mining and health sustainable development in Peru. Journal of International Management 14, 340-352.

Gray, R., Kouhy, R. y Lavers, S. (1995). Methodological themes: constructing a research database of social and environmental reporting by UK companies. Accounting, Auditing \& Accountability, Vol. 8 (2), 78-101.

Gray, R., Owen, D. y Adams, C. (1996). Accounting \& Accountability: Changes and challenges in corporate social and environmental reporting, London: Prentice Hall.

GRI (Global Reporting Initiative) (2006): Sustainability reporting guidelines. Amsterdam. GRI (Global Reporting Initiative) (2013): Sustainability reporting guidelines. Amsterdam.

Helwege, A. (2015). Challenges with resolving mining conflicts in Latin America. The Extractive Industries and Society, 2(1), 73-84.

Hernández J. (2016). Estado de la Situación de la Información de Sostenibilidad de las Empresas Peruanas. Contabilidad \& Sistemas, Volumen IX, 47-55.

Husillos, F. J. (2007). Una aproximación desde la teoría de la legitimidad a la información medioambiental revelada por las empresas españolas cotizadas. Spanish Journal of Finance and Accounting, 36(133), 97-121.

Islam, M. A., y Deegan, C. (2010). Media pressures and corporate disclosure of social responsibility performance information: a study of two global clothing and sports retail companies. Accounting and Business Research, 40(2), 131-148.

Jamali, D. (2007). The case for strategic corporate social responsibility in developing countries. Business and Society Review, 112(1), 1-27.

Jaskoski, (2014). Environmental licensing and conflict in Peru's mining sector: a path-dependent analysis. World Development, (64), 873-883.

Jenkins, H. y Yakovleva, N. (2006). Corporate social responsibility in the mining industry: Exploring trends in social and environmental disclosure. Journal of Cleaner Production, 14(3-4), 271-284.

Kemper, A. y Martin, R. (2010). After the fall: The global financial crisis as a test of corporate social responsibility theories. European Management Review, 7, 229-239.

Llena, F., Moneva, J. M. y Hernández, B. (2007). Environmental disclosures and compulsory accounting standards: the case of spanish annual reports. Business Strategy and the Environment, 16 (1),16-53.

Luken R. y Stares, R. (2005). Small business responsibility in developing countries: a threat or an opportunity? Business Strategy and the Environment, 14(1), 38-53.

Marimon, F. Alonso-Almeida, M., Rodríguez, M., y Alejandro, K. (2012). The worldwide diffusion of the global reporting initiative: what is the point?. Journal of Cleaner Production, 33, 132-144. 
Moneva, J.M. y Llena, F. (1996). Análisis de la información sobre responsabilidad social en las empresas industriales que cotizan en bolsa. Revista Española de Financiación y Contabilidad, 25(87), 361-401.

Moneva, J.M., Archel, P. y Correa C. (2006). GRI and the camouflaging of corporate unsustainability, Accounting Forum, 30 (2), 121-137.

Moneva, J.M., Acero, I. y Llena, F. (2007). Evaluación de la Información de Sostenibilidad en las Cajas de Ahorro Españolas, Cuadernos Aragoneses de Economía, 17 (1), 99-125.

Momin, M. A., y Parker, L. D. (2013). Motivations for corporate social responsibility reporting by MNC subsidiaries in an emerging country: The case of Bangladesh. The British Accounting Review, 45(3), 215-228.

Nakasone, G. (2015). Contabilidad ambiental: una propuesta basada en los reportes de sostenibilidad en las industrias minera, petrolera y de gas. Contabilidad y Negocios: Revista del Departamento Académico de Ciencias Administrativas, 10(19), 5-26.

Ortas, F. y Moneva, J.M. (2011). Origen y desarrollo de la divulgación de información de sostenibilidad: análisis del contexto Latinoamericano. Globalización, Competitividad y Gobernabilidad de Georgetown/Universia, 5(2), 16-37.

Parker, L. (2005). Social and environmental accountability research: A view from the commentary box, Accounting, Auditing and Accountability Journal, 18 (6), 842-849.

Perez-Batres, L., Miller, V. y Pisani, M. (2010). CSR, Sustainability and the Meaning of Global Reporting for Latin American Corporations. Journal of Business Ethics, 91 (2), 193-209.

Porro Rivadeneira, M. (2008). Responsabilidad social empresarial en el Perú. Revista e-mercatoria, 7(1). 1-14.

Quiroz, P. (2009). Responsabilidad social empresarial como base para el desarrollo sostenible en el Perú. Gestión Joven, (3), 6.

Rabasedas, M., Barco, M., Jara, L. (2016). Análisis intersectorial de las memorias de sostenibilidad publicadas por empresas cotizadas en Argentina. SaberEs, 8(2), 133-160.

Reverte, C. (2009). Determinants of corporate social responsibility disclosure ratings by Spanish listed firms. Journal of Business Ethics, 88(2), 351-366.

Sweeney, L. y Coughlan, J. (2008). Do different industries report Corporate Social Responsibility differently? An investigation through the lens of stakeholder theory. Journal of Marketing Communications, 14 (2), 113-124.

Slack, K. (2012). Mission impossible?: Adopting a CSR-based business model for extractive industries in developing countries. Resources Policy, 37(2), 179-184.

Tostes, M. y Chero, L. C. (2010). Análisis comparativo de la responsabilidad social en el sector financiero: Estudios de caso en Perú y Brasil 2007-2009. Derecho PUCP, (64), 299-315.

Waddock, S. (2008). Building a new institutional infrastructure for corporate responsibility. Academy of Management Perspectives, 22(3), 87-108. 